\title{
Quality Assurance, Quality Control and Method Validation in Chromatographic Applications
}

\author{
Michele L. Merves and Bruce A. Goldberger
}

University of Florida, College of Medicine, Department of Pathology, Immunology and Laboratory Medicine, P.O. Box 100275, Gainesville, FL 32610-0275, USA

\subsection{INTRODUCTION}

Clinical chemistry and toxicology are also known as clinical biochemistry or clinical pathology, and they utilize a variety of analytical procedures to analyze body fluids to aid in medical prevention, diagnosis and treatment. Analytes commonly targeted include electrolytes, creatinine, albumin and other proteins, iron and prescription (therapeutic) and illicit drugs.

The focus of this chapter is on quality assurance and quality control issues facing clinical laboratories, with emphasis on chromatographic analysis. Chromatography is a versatile analytical technique with diverse applications including gas chromatography (GC), liquid chromatography (LC), GC or LC coupled with mass spectrometry (MS) and other qualitative and quantitative bioanalytical methods.

\subsection{HISTORY}

In his book, Four Centuries of Clinical Chemistry, Rosenfeld (1999) traces the evolution of laboratory medicine from medieval times to the present. Chemical testing in biological matrices began several centuries ago, with a very crude technique for detecting pregnancy. Egyptian women would urinate on wheat and other seeds, and then observe the seeds' growth patterns to reveal pregnancy, and it was supposed, the sex of the child. Subsequently, 
Egyptians, Persians, East Indians, Chinese and other cultures observed changes in urine in order to assess disease states. Slowly, they developed a better understanding of normal and abnormal biological processes. The sixteenth and seventeenth centuries marked a major turning point in diagnostic medicine: it became clear that observation alone was not sufficient to make many diagnoses, paving the way for laboratory investigation to become an integral part of medical practice. As a result, research and clinical laboratory facilities were established, and their contributions led to fundamental technological advances in clinical laboratory medicine.

The early nineteenth century was an active time for clinical chemistry, when the role of biochemical processes in physiology began to emerge. Scientists and physicians started to realize the importance of biochemical imbalances in many diseases. However, interest in laboratory medicine languished in the middle of the nineteenth century, mostly due to the limited advancements in technology that could be applied to biochemical analyses. In its early stages, the laboratory did not provide much help to the clinician; therefore, few resources were invested in this new field. Eventually, technology would fuel a reemergence of laboratory medicine and establish its essential role in medical practice.

The term 'clinical chemical laboratory' was first used by Johann Joseph Scherer in the mid-1800s. Scherer was appointed director of the first independent hospital laboratory, in Würzburg, Germany. Although the term did not become part of the general German vocabulary for another 100 years (German-speaking areas used the term 'pathological chemistry' before 'clinical chemistry' became popular), Scherer played a key role in the development of many assays important during his career.

Medicine has advanced exponentially with diagnostic testing, and has, in turn, prompted further development in clinical chemistry and toxicology. In the late 1800s, medical technologists were trained to perform laboratory tests, eventually leading to the creation of private medical laboratory services. Eventually, it became clear that for medical laboratories to succeed, they needed to adopt specifications that would ensure the reliability of the qualitative and quantitative results they report. Quality control and assurance benefit the laboratory, because they increase productivity (Westgard and Barry, 1986) and maximize the cost-to-benefit ratio of medical laboratory testing (Kallner et al., 1999).

The impact of analytical imprecision and bias on medical decisions is mostly unknown. A large margin of error may be acceptable in some circumstances, whereas other clinical scenarios demand more accurate and precise laboratory measurements. Often, clinicians interpret laboratory results within the larger context of the patient's history and physical examination, but the influence of imprecision in laboratory data on a physician's assessment of a patient is not well documented (Kaplan, 1999). Klee et al. (1999) evaluated the effect of analytical bias on medical decisions involving three common laboratory tests: serum cholesterol testing for risk assessment of cardiac disease, serum thyroid-stimulating hormone measurement for the detection of hypothyroidism and serum prostate-specific antigen testing for prostate cancer risk assessment. In each case, the authors concluded that tighter control of analytical variability resulted in a greater certainty in patient classification.

Ensuring quality results in clinical chemistry and toxicology requires optimization of every aspect of the laboratory operation, including maintenance, waste disposal, analytical procedures, management, quality specifications, safety, cost management and overall strategies (Kallner et al., 1999). Kaplan (1999) suggested five tools that would be helpful to consider when writing guidelines for clinical laboratories: the effect of performance on specific clinical decisions, effect of performance on broad-based clinical decisions, survey of 
clinical opinions (i.e. when is an increase in error unacceptable), recommendations from professionals and societies and data from literature and proficiency testing.

Among clinical laboratories, there is a need for standardization to ensure that a result generated by one laboratory is comparable to the same measurement in another laboratory. As an example, therapeutic drug monitoring requires consistency of results over time, even when the measurements are being made in different laboratories (Groth, 1999). Several organizations are involved in establishing standards for clinical laboratory practice, including the National Academy of Clinical Biochemistry (NACB), the National Institute for Standards and Technology (NIST), the American Society of Clinical Pathologists (ASCP), the Clinical and Laboratory Standards Institute (CLSI), the International Federation of Clinical Chemistry and Laboratory Medicine (IFCC) and the International Organization for Standardization (ISO). These organizations are discussed in detail in a later section.

There has been significant progress in quality management over the last 50 years. For example, Levey and Jennings introduced statistical approaches to quality assurance in the 1950s. In the 1960s, Tonks, Barnett and Cotlove (among others) developed methods for controlling variability and established standards of quality (Westgard, 1999). In 1999, an international team of specialists with support from the International Union of Pure and Applied Chemistry (IUPAC) Clinical Chemistry Section, the International Federation of Clinical Chemistry and Laboratory Medicine (IFCC) and the World Health Organization (WHO) met for a 'consensus conference' to address laboratory quality goals (Kallner et al., 1999). Other efforts towards improving laboratory quality are national, such as the set of guidelines for bioanalytical method validation in industry in the USA that was published by the Food and Drug Administration with help from the Biopharmaceutics Coordinating Committee in the Center for Drug Evaluation and Research in cooperation with the Center for Veterinary Medicine (FDA, 2001). There are also various guidelines written for different state and local jurisdictions.

Many challenges remain in establishing universal guidelines for laboratory quality. It should be noted that universal guidelines applicable to all analytical testing within clinical chemistry and toxicology cannot be set. Testing procedures have different goals that require unique specifications. For example, a semi-quantitative analytical protocol that involves a threshold concentration may only require the use of positive and negative controls, whereas quantitative assays have stricter specifications for linearity, accuracy and precision that require the use of multiple calibrators and controls.

\subsection{DEFINITION OF QUALITY ASSURANCE AND QUALITY CONTROL}

Quality assurance and quality control procedures are vital aspects of clinical chemistry and toxicology (Garfield et al. 2000; Tilstone, 2000; Ratliff, 2003). The terms quality assurance and quality control sometimes are used interchangeably, but the two terms have different meanings and should be used in different contexts. Quality assurance (QA) encompasses quality control, but also applies to virtually all aspects of laboratory operation. QA requires that all laboratory procedures are documented. Systematic internal and external review of these practices and documentation are essential to achieving optimal efficiency and accuracy (Rosenfeld, 1999). Key aspects of the QA process are laboratory accreditation, proficiency testing and staff education. Depending on the jurisdiction, there are many different types of 
guidelines under the umbrella of QA. For example, the laboratories or technologists may be required to be certified by the state or other agencies. In addition, continuing staff education is essential for maintaining and updating credentials, and proficiency testing may be required in order to maintain certification.

Quality control (QC) originated from the industrial goal of producing each batch of product with consistently high quality, detecting and correcting process errors that result in defective products. Therefore, QC is an essential component of a laboratory QA program, because it provides a mechanism for monitoring the quality of laboratory results. To ensure that a set of analytical results meets the desired specifications, control specimens are included in each step of the procedure. These controls should include both normal and abnormal concentrations of analyte (Goldberger et al., 1997).

\subsection{PROFESSIONAL ORGANIZATIONS}

As mentioned in Section 1.2, various professional organizations are involved in establishing standards for clinical laboratory practices and key organizations include the NACB, NIST, ASCP, CLSI, IFCC and ISO.

The National Academy of Clinical Biochemistry (NACB) is the Academy of the American Association for Clinical Chemistry (AACC). Its mission is 'to elevate the science and practice of clinical laboratory medicine by promoting research, education and professional development in clinical biochemistry and by serving as the leading scientific advisory group to AACC and other organizations' (http://www.nacb.org). It has six objectives which promote research, education, professional development, scientific advisory roles, recognition and infrastructure. Since the mid-1990s, the NACB has published several consensus-based laboratory medicine practice guidelines (LMPGs).

The National Institute for Standards and Technology (NIST) was established in 1901 as the National Bureau of Standards. The mission of NIST is 'to promote US innovation and industrial competitiveness by advancing measurement science, standards and technology in ways that enhance economic security and improve our quality of life' (http://www.nist.gov). The principal programs include NIST Laboratories, the Baldrige National Quality Program, the Manufacturing Extension Partnership and the Advanced Technology Program. As part of its commitment to the advancement of technology, NIST produces reference materials for bioanalysis. These standard reference materials are essential for validating the accuracy of analytical methods.

The American Society of Clinical Pathologists (ASCP) was founded in 1922 and its mission is to 'provide excellence in education, certification, and advocacy on behalf of patients, pathologists, and laboratory professionals' (http://www.ascp.org). The ASCP has developed many educational programs to advance the role of the laboratory in medical diagnosis (Rosenfeld, 1999).

In 1968, the National Committee for Clinical Laboratory Standards was established, and recently changed its name to the Clinical and Laboratory Standards Institute (CLSI) (http:// www.nccls.org). The CLSI is a nonprofit organization that sponsors educational programs in order to promote national and international standards for clinical laboratories. This is a noteworthy task, especially since following these specifications is not mandatory and no enforcement agency exists to ensure each laboratory follows the CLSI guidelines. As part of its educational programs, the CLSI publishes consensus documents on laboratory 
procedures, reference methods and evaluation protocols. A major goal of CSLI is to encourage high quality in the laboratory in order to maximize patient care benefits (Rosenfeld, 1999).

The International Federation of Clinical Chemistry and Laboratory Medicine (IFCC) was established in the mid-1950s with goals to 'advance knowledge and promote the interests of biochemistry in its clinical (medical) aspects' (www.ifcc.org). IFCC was originally formed under IUPAC, but became independent in the late 1960s. However, it has maintained a close affiliation with IUPAC, and also WHO. Aside from its role in publishing guidelines and maintaining educational opportunities for its members, the IFCC plays a key role in developing national societies within countries without such resources.

The International Organization for Standardization (ISO) is a worldwide organization representing national standard societies from approximately 149 countries. ISO began in 1947 after a delegation of 25 countries met with the desire to 'facilitate the international coordination and unification of industrial standards' (http://www.iso.org). The ISO mission is to promote global standardization for exchange of services among nations. ISO publishes International Standards, which encourage intellectual, scientific, technological and economic exchange. For example, ISO 9000 is a set of four documents (9001-9004) that provide standards based on an international consensus for various aspects of quality assurance. In addition, ISO organizes Technical Advisory Groups to study quality control and assurance issues (Burtis and Ashwood, 2001).

\subsection{INTERNAL QUALITY ASSURANCE AND CONTROL}

Internal quality assurance and control procedures are designed to guarantee the reliability of the results generated by the laboratory; QA and QC ensure that the reported results contain the appropriate accuracy and precision through stringent monitoring of the analytical process, documentation of procedures and method validation.

\subsubsection{Standard operating procedure manual}

The standard operating procedure (SOP) manual contains the procedures validated by the laboratory; it is a complete set of instructions for pre-analytical, analytical and postanalytical methodology and also procedures for quality assurance/control, chain-of-custody and security. Each step in the handling of the specimen should be evaluated, optimized where possible and documented in the SOP. Important steps in the analytical process include collection, transport and accessioning of the specimen, sample preparation, isolation and detection of the analytes, production of the report and disposal of the specimen. This chapter focuses on the quality assurance and control issues for analytical method development and validation as well as statistical representation of the data.

\subsubsection{Method development}

Analytical methods are developed to meet specific needs. For example, pharmacodynamic and pharmacokinetic assessment of a new drug will require the ability to measure it in 
biological matrices. These methods should be developed under the 'Good Laboratory Practice' (GLP) standards issued by the US Food and Drug Administration (FDA), and provide accurate and precise results. Typically, constraints are placed on the new methods, such as pre-established administrative reporting limits (e.g. cut-off concentrations) or existing QA/QC requirements (Goldberger et al., 1997). Therefore, the intent and analytical goals must be established for the new protocol before the analytical technique is optimized on the bench. The analytical goals (also known as analytical quality specifications, performance standards or performance goals) are required not only for method development and validation, but also to help justify the purchase of new instrumentation and assist manufacturers in the design, construction and marketing of new equipment and reagents. In addition, the analytical goals provide specifications for relevant external quality assurance programs (Fraser et al., 1999).

\subsubsection{Method validation}

Once a new analytical method has been developed, it must be optimized and standardized to meet the purposes for which it was developed. Validation studies confirm that a new assay has met its required performance specifications, and must be done before the analytical procedure can become a routine laboratory protocol. There are multiple approaches to validating analytical methods. The most common is to compare results from the new method with results obtained using an established, or reference, assay. Alternatively, standard reference materials (e.g. standards certified by NIST) can be analyzed by the new method to demonstrate its accuracy. When available, authentic specimens should be tested and compared against a previously validated protocol.

Each analytical method will have a different purpose and therefore different analytical performance requirements. Some of the performance parameters are accuracy and precision, recovery, limits of detection (sensitivity) and quantitation, range of linearity, specificity, stability, carryover potential and ruggedness. Other methadological factors are selection of a reference standard, internal standard, derivatizing agent, ions for selected-ion monitoring in mass spectrometry, instrumentation and chromatographic performance (Goldberger et al., 1997; Gerhards et al., 1999; Wu et al., 1999; Garfield et al., 2000; Burtis and Ashwood, 2001; FDA, 2001; Jiménez et al., 2002). These parameters can be assessed within the same batch (intra-assay, within-run) or between batches (inter-assay, between-run or day-to-day) using reference standards and quality control samples. Most standards and quality control materials have been verified and are available commercially, often in the form as lyophilized or liquid samples.

Accuracy and precision are the most important characteristics of an analytical method; they give the best indication of random and systematic error associated with the analytical measurement. Systematic error refers to the deviation of an analytical result from the 'true' value, and therefore affects the accuracy of a method. One the other hand, random errors influence the precision of a method (Kallner et al., 1999). Ideally, accuracy and precision should be assessed at multiple concentrations within the linear range of the assay (low, medium and high concentration).

After a method has been validated, it can be used for routine laboratory analyses. There may be additional analytical considerations that are important in routine assays. Analysis of the specimens should be done in a timely manner, especially when the analyte is unstable. 
Generally, specimens can be run singly unless duplicate measurements are required in order to improve precision and reliability. Quality controls should be included with each batch of specimens and the QC results monitored to detect trends in analytical performance that can potentially affect the accuracy of results.

\subsubsection{Accuracy}

Accuracy defines how closely the measured concentration agrees with the true (fortified) value. For most analytical applications, laboratories should achieve a level of accuracy within $5-10 \%$ of certified concentrations in reference materials. Accuracy is often expressed as a percentage difference from the true value:

$$
\operatorname{Accuracy}(\%)=[(\text { mean }- \text { true value }) / \text { true value }] \times 100
$$

Accuracy can also be expressed as the percentage of the mean to the true value:

$$
\operatorname{Accuracy}(\%)=(\text { mean/true value }) \times 100
$$

Ideally, accuracy should be determined at multiple concentrations of the analyte. Since repeated measurements by any analytical method will produce a Gaussian distribution of results, Student's $t$-test provides a statistical way to assess the agreement of measured values with the target or 'true' value.

\subsubsection{Precision}

Precision describes the variability in a set of measurements, i.e. how closely repeated measurements on a single sample agree. Precision can be calculated for samples within an individual run (intra-assay precision) or across multiple runs (inter-assay precision). Precision is customarily expressed as the percentage coefficient of variation $(\% \mathrm{CV}$, or simply $\mathrm{CV}$ ), which is the standard deviation expressed as a percentage of the mean:

$$
\mathrm{CV}=(\text { standard deviation } / \text { mean }) \times 100
$$

Typically, laboratories should achieve a level of precision of $<10 \%$; however, in some instances, the CV may be $>10 \%$, especially at or near the lower limit of quantitation, when background noise is a larger portion of the total signal measured. The CV should be determined at multiple concentrations of the analyte.

\subsubsection{Recovery}

When a protocol requires some manipulation of the specimen prior to analysis, such as an extraction step, there may be opportunities for the analyte concentration to be diminished due to pre-analytical loss. Therefore, it may be important to calculate the recovery of the analyte. Analyte recovery can be calculated by comparing the detector response from the 
analyte after it has been extracted to the detector response from an unextracted sample. The unextracted sample represents $100 \%$ recovery because it contains the 'true' concentration of the analyte. It is not necessary to achieve $100 \%$ recovery, but the recovery should be reproducible and evaluated at three separate concentrations (FDA, 2001).

\subsubsection{Lower limits of detection (sensitivity) and quantitation}

The lower limit of detection (LLOD) and the lower limit of quantitation (LLOQ) are helpful parameters when validating an analytical method. The LLOD is the lowest concentration of the analyte that can be detected. Customarily, the LLOD corresponds to the concentration of analyte producing a signal at least three times greater than the analytical background noise. Establishing an LLOD requires knowing the signal-to-noise ratio, acceptable chromatographic separation, predicted retention time and, for mass spectrometric (MS) applications, acceptable $m / z$ ion ratios.

The LLOQ is the lowest concentration of the analyte that is detected with a specified accuracy and precision. In practice, the LLOQ often corresponds to a signal 5-10 times greater than the analytical background noise. As an example, the LLOQ might correspond to the concentration meeting all of the LLOD criteria, be quantified within $10 \%$ of the target concentration and produce a $\mathrm{CV}$ of $<10 \%$. These parameters can be optimized by manipulating several factors, including the specimen volume, the detector threshold (gain), the type and condition of the chromatographic column, preconcentration of the analyte, the amount and type of internal standard, the extraction efficiency and the analytical method (Goldberger et al., 1997).

\subsubsection{Range of linearity}

Most, though not all, analytical methods produce results that vary linearly with analyte concentration, so the term calibration 'curve' is something of a misnomer. The range of linearity of an analytical method is determined by measuring standard solutions at multiple concentrations (calibrators), sometimes using an internal or external standard for quantitation. Calibrators should be prepared in a matrix similar to authentic specimens, to compensate for potential matrix effects. The practical linear range, which encompasses the expected concentrations in authentic specimens, should be assessed, i.e. challenged at its upper and lower limits, each time the assay is run. An acceptable linear range depends on various criteria, such as chromatographic resolution, correct retention time, appropriate $\mathrm{m} / \mathrm{z}$ ion ratios and a correlation coefficient $(r)$ or coefficient of determination $\left(R^{2}\right)>0.99$ (Goldberger et al., 1997).

There are various ways to derive a calibration curve. Multi-point calibration curves, for example, include a minimum of three different concentrations of the analyte. For semiquantitative assays, a single-point calibration is common. The single point is usually the threshold concentration used to determine whether a specimen is positive or negative for the analyte of interest. Depending on the validation process and performance characteristics of the assay, a single-point calibration curve may also be used in quantitative applications over a limited range of linearity. A historical (pre-established) multi-point calibration curve may also be used, but only if the stability of the analytical method over time has been well established (Goldberger et al., 1997). 


\subsubsection{Specificity}

Specificity, or selectivity, is defined as the capability of the methodology to identify the analyte of interest in the presence of matrix components and potentially interfering substances (FDA, 2001; Goldberger et al., 1997). Analytical interferences include endogenous matrix components, decomposition products, metabolites and other structurally related compounds. FDA guidelines (FDA, 2001) suggest that six blank specimens from different sources be analyzed for matrix effects. Fewer samples may be required when using methodology with MS detection.

In contrast, there are instances in which decreased specificity is beneficial, such as in assays designed to detect an entire class of compounds. For example, antibody crossreactivity is essential in immunoassays used to screen for multiple drugs or drug classes. Screening methods ordinarily are paired with confirmatory testing methods that use a different analytical methodology with increased specificity.

\subsubsection{Stability}

Some analytes are not stable under certain transport, storage and analytical conditions. Therefore, it is essential to evaluate the stability of an analyte under a variety of conditions. Specimens may be collected with preservatives (e.g. sodium fluoride) and stored refrigerated or frozen. During analysis, the addition of an internal standard and/or the use of quality control samples can be helpful in evaluating the stability of an analyte during the entire analytical procedure.

For assays requiring specimens to be frozen, the stability of the analyte in a freeze-thaw cycle should be validated. FDA guidelines (FDA, 2001) suggest that analytes should be tested in triplicate over three freeze-thaw cycles at two different concentrations.

\subsubsection{Carryover}

Carryover is defined as the 'contamination of a sample by a sample analyzed immediately prior to it' (Goldberger et al., 1997). Therefore, the validation process should assess carryover potential in highly concentrated specimens and the acceptable limit of carryover into otherwise negative samples. Suggested ways to minimize carryover include the use of solvent washes after each injection, the use of solvent blanks between cases, dilution of specimens prior to extraction, dilution of extracts prior to injection, occasional reassessment of the minimum carryover limit and injection of specimen extracts in ascending concentration order (determined by initial screening). Carryover is most often due to contamination by the sampling syringe exposed to the previous specimen. Therefore, when carryover is suspected, the specimen in question should be re-extracted, since re-injection from the already contaminated vial will produce the same carryover error.

\subsubsection{Ruggedness}

Ruggedness refers to the ability of an analytical method to withstand minor procedural changes without affecting the overall performance of the assay. The ruggedness of an assay 
can be very important, particularly when multiple technicians or multiple laboratories perform the same procedure. Therefore, it is useful to determine the critical steps in the assay, such as $\mathrm{pH}$, composition of solvent mixtures, derivatizing chemicals, temperature and incubation times (Goldberger et al., 1997). In addition, specimen volume may be critical, so any adjustments to the volume of specimen should be validated. If specimen dilutions are required, the preferred diluent is blank (analyte-free) matrix (FDA, 2001).

\subsubsection{Selection of a reference standard}

Selection of a reference standard for most analytical methods is a straightforward task, involving acquisition of the relevant analyte in a state of known composition and purity. However, some analytes are not available in pure form, and structurally similar analogs (e.g. free base) may be used instead. Standard materials should be of a known purity, and the source, lot number, expiration date and certification of identity should be recorded (FDA, 2001).

\subsubsection{Selection of an internal standard and standard addition}

Analytes may be quantitated using an internal standard or by the method of standard addition. An internal standard is a structural analog (e.g. deuterium-labeled compound), a known amount of which is added to specimens prior to any preparatory steps such as extraction or hydrolysis. Ideally, the internal standard should be as chemically similar to the analyte of interest as possible, so that throughout the analytical procedure (e.g. extraction, chromatographic separation and ionization processes) and under the specified conditions (e.g. derivatization), its recovery will be similar to the analyte's. The concentration of analyte can then be determined from the ratio of the analyte response to the internal standard response.

The addition of an internal standard is advantageous because detection of the internal standard ensures that the extraction step successfully recovered analyte (if present). Any losses associated with preparatory steps are not critical, because this procedure relies on the ratio of concentrations, not absolute concentration.

Standard addition can be an alternative method for the quantitation of analytes. The general concept is to add known concentrations of the analyte to multiple aliquots of the unknown specimen. By extrapolation, the original concentration of the (unfortified) specimen can be determined. This technique is particularly helpful when a calibration curve cannot be generated using standards in a similar matrix.

\subsubsection{Selection of derivatization agent}

In the context of chromatographic applications, derivatization is the process of chemically altering a compound with the intent to improve the sensitivity with which it can be detected. Often, derivatization involves reacting polar groups (-NH, - $\mathrm{OH}$ and $-\mathrm{SH}$ ) with perfluoryl or silyl derivatization reagents to increase the volatility of the compound. Some derivatives enhance the detector response, improving sensitivity. In other circumstances, it may be 
beneficial to increase the mass of a compound to distinguish its ions from background noise or similar structures (Goldberger et al., 1997). There are some of disadvantages to derivatization, such as requiring an extra step (often manual) in the analytical method and the absence of many derivatized products in commercial mass spectral libraries. Therefore, laboratories may need to produce their own reference libraries when using unusual derivatives for MS applications.

\subsubsection{Selection of ions for selected-ion monitoring or full-scan analysis}

Assays that utilize MS require the selection of ions that uniquely represent the analyte and internal standard without any potential interferences (Goldberger et al., 1997). It is helpful to identify ions for both quantitative and qualitative purposes. Ratios between the ions can be helpful to confirm the compound of interest. The base peak (ion with the highest abundance in the mass spectrum) is often preferred as the quantitative ion because it allows for the greatest sensitivity.

\subsubsection{Chromatographic performance}

Methods involving chromatographic separations prior to detection require optimization of the chromatographic conditions for best performance. A Gaussian peak shape (no tailing or fronting), resolution (baseline separation from nearby peaks) and reproducible retention time are necessary for identification of the analytes of interest (Goldberger et al., 1997).

\subsubsection{Statistical evaluation of quality control}

Proper interpretation of quality control data requires presentation in a format that makes systematic bias readily apparent. Based on the work of Shewhart, Levey and Jennings, Westgard and co-workers proposed in 1981, an algorithm for monitoring quality control in clinical laboratories (Westgard et al., 1981). 'Westgard's Rules' provide guidance for determining when control values are acceptable or, alternatively, when control measurements reflect changes in the performance of the analytical method (Westgard and Barry, 1986; Burtis and Ashwood, 2001).

In a Levey-Jennings control chart, the $x$-axis represents time or number of analytical runs and the $y$-axis represents the control results (Figure 1.1a). Control values are plotted on the Levey-Jennings chart, and should fall within a statistically predicted range, based on a Gaussian distribution. The acceptable range of values is typically indicated by lines on the $y$-axis representing the upper and lower limits for control results (commonly \pm 2 standard deviations or defined percentages from the mean control value). Westgard described a multirule procedure using the Levey-Jennings chart to detect deviations from Gaussian behavior that reflect analytical bias (Burtis and Ashwood, 2001).

Another way to present control data is the cumulative sum control chart. In this method, the $x$-axis is either time or number of control observations and the $y$-axis is the cumulative sum (cusum), or the difference between the measured control value and the predicted mean 


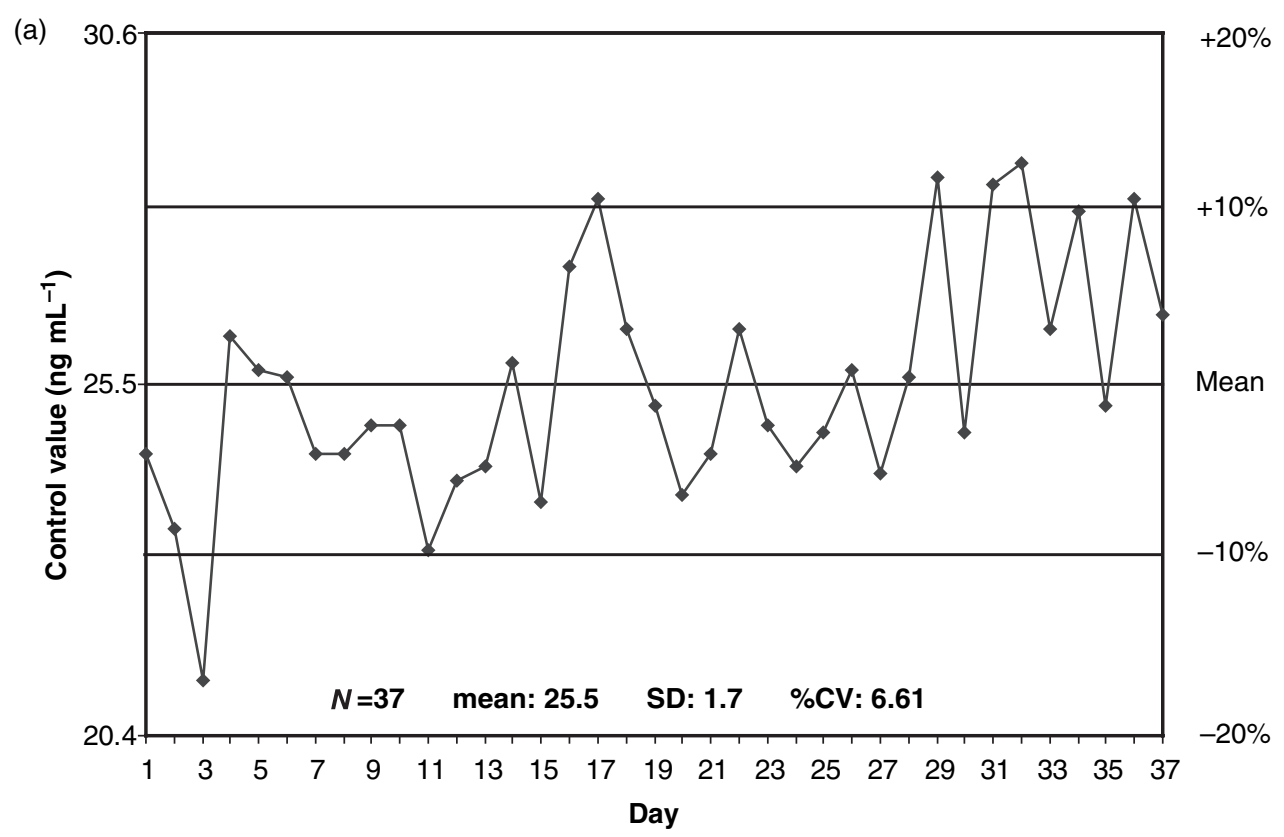

(b)

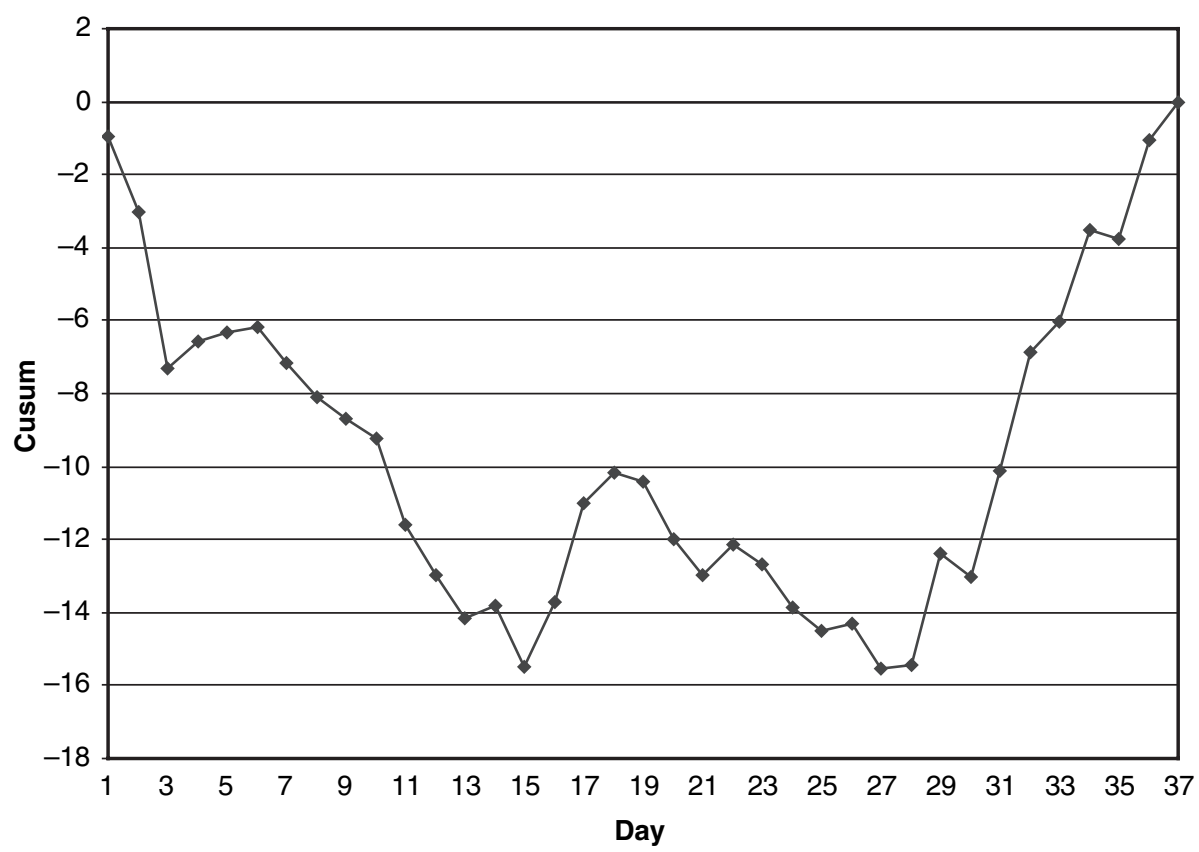

Figure 1.1 Examples of (a) Levey-Jennings and (b) cumulative sum (cusum) control charts using inter-assay quality control data from an alprazolam GC-MS assay. Since the cusum chart persents the cumulative sum of deviations from the mean, it is more sensitive to small biases that develop over time, whereas Levey-Jennings charts are most useful for detecting changes in the precision of the assay 
concentration of the control material (Figure 1.1b). The cusum is calculated by adding this difference to the differences from previous control observations. Random errors should produce cusum values that cross the zero line (mean value) multiple times. However, if there are other sources of error, then the cusum will deviate from the mean value in one or the other direction. Other quality control charts, such as mean, standard deviation and range charts, are used in industry, but are not common in clinical chemistry and toxicology (Westgard and Barry, 1986).

\subsection{EXTERNAL QUALITY ASSURANCE}

External quality assurance (EQA) is fundamental to the standardization of clinical laboratory methods because it provides a means to compare results generated in one laboratory with those of peer laboratories subscribing to the same EQA program. EQA programs are especially beneficial since internal QA and QC procedures are limited in their ability to detect bias in analytical methods. Internal QA/QC can only detect errors that result in a deviation from the original method validation: inherent errors in the method may go unnoticed. Therefore, it is helpful to compare the results produced by a new method with those from other laboratories (Burtis and Ashwood, 2001). Monitoring the performance of laboratory procedures in a consistent manner keeps the laboratory accountable, and can reveal systematic errors that would otherwise be undetected. A prominent component of EQA is proficiency testing.

Proficiency testing in clinical laboratories began in the late 1940s as a response to lack of reproducibility among laboratories (Rosenfeld, 1999). In 1947, Belk and Sunderman published the results of a study in which they surveyed clinical laboratories in Pennsylvania for accuracy among common chemical testing procedures in hospital laboratories (Sunderman, 1992). Thereafter, other states conducted similar studies, and eventually monthly samples were mailed to various laboratories worldwide and the statistical summaries were returned to these laboratories, comparing their results with other laboratories performing the same analyses. The Sunderman Proficiency Testing Service diligently continued this program for 36 years until 1985, when the American Society of Clinical Pathologists took over the service. Since then, many other organizations, such as College of American Pathologists, the American Association of Bioanalysts and American Association for Clinical Chemistry have provided various forms of proficiency testing. Combined, these external testing procedures have helped to standardize testing among laboratories and enhance the overall quality of laboratory practices.

\section{REFERENCES}

Burtis, C. A., Ashwood, E. R. (Eds) (2001). Tietz Fundamentals of Clinical Chemistry, 5th edn. Philadelphia, PA: W. B. Saunders.

FDA (2001). Guidance for Industry: Bioanalytical Method Validation. US Department of Health and Human Services, Food and Drug Administration, Center for Drug Evaluation and Research (CDER), Center for Veterinary Medicine (CVM). http://www.fda.gov/cder/guidance/4252fnl.pdf.

Fraser, C. G., Kallner, A., Kenny, D., Hyltoft Petersen, P. (1999). Introduction: strategies to set global quality specifications in laboratory medicine. Scand J Clin Lab Invest, 59, 477-478. 
Garfield, F. M., Klesta, E., Hirsch, J. (2000). Quality Assurance Principles for Analytical Laboratories, 3rd edn. Gaithersburg, MD: AOAC International.

Gerhards, P., Bons, U., Sawazki, J., Szigan, J., Wertmann, A. (1999). GC/MS in Clinical Chemistry. New York: Wiley-VCH.

Goldberger, B. A., Huestis, M. A., Wilkins, D. G. (1997). Commonly practiced quality control and quality assurance procedures for gas chromatography/mass spectrometry analysis in forensic urine drug-testing laboratories. Forensic Sci Rev, 9, 59-80.

Groth, T. (1999). Series analyses and quality specifications required for monitoring over time. Scand $J$ Clin Lab Invest, 59, 501-508.

Jiménez, C., Ventura, R., Segura, J. (2002). Validation of qualitative chromatographic methods: strategy in antidoping control laboratories. J Chromatogr B, 767, 341-351.

Kallner, A., McQueen, M., Heuck, C. (1999). The Stockholm Consensus Conference on quality specifications in laboratory medicine, 25-26 April 1999. Scand J Clin Lab Invest, 59, 475-585.

Kaplan, L. A. (1999). Determination and application of desirable analytical performance goals: the ISO/ TC 212 approach. Scand J Clin Lab Invest, 59, 479-482.

Klee, G. G., Schryver, P. G., Kisabeth, R. M. (1999). Analytic bias specifications based on the analysis of effects on performance of medical guidelines. Scand J Clin Lab Invest, 59, 509-512.

Ratliff, T. A. (2003). Laboratory Quality Assurance System: a Manual of Quality Procedures and Forms, 3rd edn. Hoboken, NJ, John Wiley \& Sons, Inc.

Rosenfeld, L. (1999). Four Centuries of Clinical Chemistry. Amsterdam: Gordon and Breach.

Sunderman, F. W. Sr. (1992). The history of proficiency testing/quality control. Clin Chem, 38, 12051209.

Tilstone, W. J. (2000). Quality assurance. In Encyclopedia of Forensic Sciences, J. A. Siegel, P. J. Saukko, G. C. Knupfer, G. C. (Eds). San Diego, CA: Academic Press, pp. 1307-1314.

Westgard, J. O. (1999). The need for a system of quality standards for modern quality management. Scand J Clin Lab Invest, 59, 483-486.

Westgard, J. O., Barry, P. L. (1986). Cost-effective Quality Control: Managing the Quality and Productivity of Analytical Processes. Washington, DC: AACC Press.

Westgard, J. O., Barry, P. L., Hunt, M. R., Groth, T. (1981). A multi-rule Shewhart chart for quality control in clinical chemistry. Clin Chem, 27, 493-501.

Wu, A. H. B., Hill, D. W., Crouch, D., Hodnett, C. N., McCurdy, H. H. (1999). Minimal standards for the performance and interpretation of toxicology tests in legal proceedings. J Forensic Sci, 44, 516-522. 